\title{
A numerical scheme for the solution of neutral integro-differential equations including variable delay
}

\author{
Burcu Gürbüz ${ }^{1,2}$ (D)
}

Received: 3 August 2020 / Accepted: 17 February 2021 / Published online: 13 March 2021

(c) The Author(s) 2021

\begin{abstract}
In this study, an effective numerical technique has been introduced for finding the solutions of the first-order integro-differential equations including neutral terms with variable delays. The problem has been defined by using the neutral integrodifferential equations with initial value. Then, an alternative numerical method has been introduced for solving these type of problems. The method is expressed by fundamental matrices, Laguerre polynomials with their matrix forms. Besides, the solution has been obtained by using the collocation points with regard to the reduced system of algebraic equations and Laguerre series.
\end{abstract}

Keywords Neutral type equations $\cdot$ Integro-differential equation $\cdot$ Variable delays $\cdot$ Laguerre polynomial and series $\cdot$ Matrix method

\section{Introduction}

Delay differential equations in neutral type and integro-differential equations are of an attractive interest in many applications in science and engineering. In applied mathematics, they have an increasing enthusiasm by their implementation in dynamical systems, electrodynamics and mechanics as well. In recent years, numerical treatment of the neutral type integro-differential equations has been arised [1]. These type of equations occur in mechanics, physics, technical problems such as progress for showing cutting and infeed grinding. Besides, they describe some procedures in chemistry and physics for reactors. Moreover, some well-known biological processes growth, death and birth are determined by neutral type equations. In ecological phenomena, some models with respect to the neutral type integro-differential equations are used for evolution equations of single species [2]. Furthermore, many applications of these types of equations exist in medicine. For instance, sugar quantity in blood is modeled by using them; immunology, epidemiology, cancer chemotherapy may explain different aspects of human

Burcu Gürbüz

burcu.gurbuz@uni-mainz.de

Johannes Gutenberg - University of Mainz, Mainz, Germany

2 Present Address: Institute of Mathematics, Johannes Gutenberg-University Mainz, IMainz, Germany body interaction with diseases. As another example, in Fig. 1 model of pressure regulation of model of arterial blood is represented by the formulation of functional differential equations. In this figure, arterial vessels are shown by $A$ and $B$. These arterial vessels have connections to each other, blood flow from $A$ to $B$ has a rate which is shown by $Q, R$ represents peripheral resistance, and the heart productivity is denoted by $Q_{\mathrm{h}}$. The incoming liquid rate is $Q_{\text {in }}$ while the outcoming rate is $Q_{\text {out }}$ [3]. Furthermore, epidemic of the human immunodeficiency virus (HIV) is modeled by the system of functional integro-differential equations. Besides, fishing process, river pollution control can be described and these examples are ecological applications in the ecology field.

Neutral type delay integro-differential equations under the initial condition are of numerical solutions which have been reached by many authors. Such problems have difficulties in motivation but also often appear at numerical investigations. Functional integro-differential equations including Volterra type integrals have been searched with regard to numerical analysis aspect by Brunner [4]. Collocation methods have been applied for solving Volterra functional integral equations including non-vanishing delays [5]. Besides, in the literature, continuous spline collocation methods [6], Lagrange interpolation and Chebyshev interpolation [7], Adams-Moulton method [8], backward substitution method [9], continuous Runge-Kutta method [10-12], Spectral method [13] have been implemented in order to find 


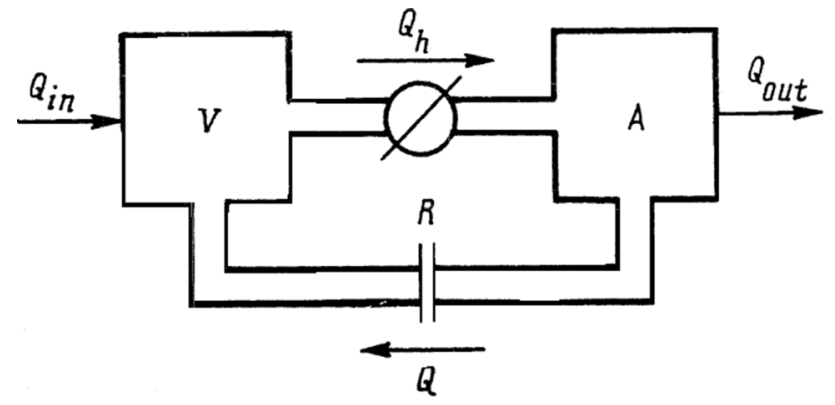

Fig. 1 A diagram to describe the cardiovascular system discretization as a reliable tool. On the other hand, Laguerre polynomials give powerful solutions, especially on the positive interval since its applications in several fields with these types of properties.

\section{Fundamental relations}

In here, the matrix forms of Eq. (1) are composed. First of all, it is organized as

$D_{3}+D_{2}=D_{1}+I+G$

where

$D_{1}=P_{0}(t) y(t) ; \quad D_{2}=P_{1}(t) y^{\prime}(t-\tau(t)) ; \quad D_{3}=y^{\prime}(t) ; \quad I=\int_{t-u(t)}^{t-v(t)} K(t, s) y(s) \mathrm{d} s ; \quad G=g(t)$.

the solutions of neutral type integro-differential equations including variable delays.

In this work, the following first-order integro-differential equation including neutral terms and variable delays is considered as

$y^{\prime}(t)+P_{1}(t) y^{\prime}(t-\tau(t))=P_{0}(t) y(t)+\int_{t-u(t)}^{t-v(t)} K(t, s) y(s) \mathrm{d} s+g(t), \quad 0 \leq t, s \leq b$,

under the initial condition

$y^{\prime}(0)=\lambda_{0}$,

where $P_{0}(t), P_{1}(t), u(t), v(t), g(t)$ and the delay term $\tau(t)$ are defined as continuous functions for $\infty>b>t \geq 0$. Here the aim is about finding a numerical solution $y_{N}(t)$ by using the truncated Laguerre series of given problem (1)-(2):

$y_{N}(t)=\sum_{n=0}^{N} a_{n} L_{n}(t)$

where $a_{n}, n=0,1, \ldots, N$ are unknown coefficients; $L_{n}(t)$ are the Laguerre polynomials for $n=0,1, \ldots, N$ and defined as

$L_{n}(t)=\sum_{r=0}^{n} \frac{(-1)^{r}}{r !}\left(\begin{array}{l}n \\ r\end{array}\right) t^{r}$

\section{Numerical method}

In this section, the numerical method based on Laguerre polynomials is introduced. The main advantage of the method lies in its straightforwardness since it has no aim for
Then, the matrix form of the numerical solution (3) is considered as

$[y(t)]=\mathbf{L}(t) \mathbf{A}$,

where

$\mathbf{L}(t)=\left[\begin{array}{lllll}L_{0}(t) & L_{1}(t) & L_{2}(t) & \ldots & L_{N}(t)\end{array}\right]$ and $\mathbf{A}=\left[\begin{array}{llll}a_{0} & a_{1} & \ldots & a_{N}\end{array}\right]^{T}$.

here $\mathbf{L}(t)$ is defined as in the matrix form:

$\mathbf{L}(t)=\mathbf{X}(t) \mathbf{H}$

where

$\mathbf{X}(t)=\left[\begin{array}{lllll}1 & t & t^{2} & \ldots & t^{N}\end{array}\right]$

$\mathbf{H}=\left[\begin{array}{ccccc}\frac{(-1)^{0}}{0 !}\left(\begin{array}{l}0 \\ 0\end{array}\right) & \frac{(-1)^{0}}{0 !}\left(\begin{array}{l}1 \\ 0\end{array}\right) & \frac{(-1)^{0}}{0 !}\left(\begin{array}{l}2 \\ 0\end{array}\right) & \cdots & \frac{(-1)^{0}}{0 !}\left(\begin{array}{c}N \\ 0\end{array}\right) \\ 0 & \frac{(-1)^{1}}{1 !}\left(\begin{array}{l}1 \\ 1\end{array}\right) & \frac{(-1)^{1}}{1 !}\left(\begin{array}{l}2 \\ 1\end{array}\right) & \cdots & \frac{(-1)^{1}}{1 !}\left(\begin{array}{c}N \\ 1\end{array}\right) \\ 0 & 0 & \frac{(-1)^{2}}{2 !}\left(\begin{array}{c}2 \\ 2\end{array}\right) & \cdots & \frac{(-1)^{2}}{2 !}\left(\begin{array}{c}N \\ 2\end{array}\right) \\ \vdots & \vdots & \vdots & \ddots & \vdots \\ 0 & 0 & 0 & \cdots & \frac{(-1)^{N}}{N !}\left(\begin{array}{c}N \\ N\end{array}\right)\end{array}\right]$

Thus, the connection between $\mathbf{X}(t)$ and $\mathbf{X}^{\prime}(t)$ is defined as

$\mathbf{X}^{\prime}(t)=\mathbf{X}(t) \mathbf{B}$ 


$$
\int_{t-u(t)}^{t-v(t)} \mathbf{X}(t) \mathbf{K} \mathbf{X}^{T}(s) \mathbf{L}(s) \mathbf{A} d s=\mathbf{X}(t) \mathbf{K} \int_{t-u(t)}^{t-v(t)} \mathbf{X}^{T}(s) \mathbf{X}(s) d s \mathbf{H A}=\mathbf{X}(t) \mathbf{K} \mathbf{Q}(t) \mathbf{H A},
$$

$\mathbf{B}=\left[\begin{array}{ccccc}0 & 1 & 0 & \ldots & 0 \\ 0 & 0 & 2 & \ldots & 0 \\ \vdots & \vdots & \vdots & \ddots & \vdots \\ 0 & 0 & 0 & N & 0 \\ 0 & 0 & 0 & 0 & 0\end{array}\right]$

Therefore, the matrix relations (7) and (8) are used and $\mathbf{L}^{\prime}(t)$ is defined as follows

$\mathbf{L}^{\prime}(t)=\mathbf{X}(t) \mathbf{B H}$

So that, from (7) and (9)

$\mathbf{X}(t)=\mathbf{L}(t) \mathbf{H}^{-1} ; \mathbf{L}^{\prime}(t)=\mathbf{L}(t) \mathbf{C}$,

is obtained in which $\mathbf{C}$ is described as

$\mathbf{C}=\left[c_{p q}\right], \quad c_{p q}=\left\{\begin{array}{c}-1, p<q \\ 0, p \geq q\end{array}\right.$.

Thus, from (6), (7), and (10) [14]:

$\left[y^{\prime}(t)\right]=\mathbf{L}(t) \mathbf{C A}$

By replacing $t \rightarrow t-\tau(t)$ into (11) and by using (9), we obtain

$\left[y^{\prime}(t-\tau(t))\right]=\mathbf{L}(t-\tau(t)) \mathbf{C A}=\mathbf{X}(t-\tau(t)) \mathbf{B H A}=\mathbf{X}(t) \mathbf{T}(t-\tau(t)) \mathbf{B H A}$ where

$\mathbf{Q}(t)=\int_{t-u(t)}^{t-v(t)} \mathbf{X}^{T}(s) \mathbf{X}(s) d s=\int_{t-u(t)}^{t-v(t)}\left[s^{i+j}\right] d s=\left[q_{i j}(t)\right]$,

and

$q_{i j}(t)=\frac{(t-v(t))^{i+j+1}-(t-u(t))^{i+j+1}}{i+j+1}, \quad i, j=0,1, \ldots, N$.

Similarly, from (11), the initial condition in Eq. (2) has the matrix form as

$\left[y^{\prime}(0)\right]=\mathbf{L}(0) \mathbf{C A}=\left[\lambda_{0}\right]$.

Therefore, we obtain the matrix forms of $D_{1}, D_{2}, D_{3}$ and $I$ in Eq. (5), from (6), (12), (11), and (14), respectively, as

$D_{1}=\mathbf{P}_{0}(t) \mathbf{L}(t) \mathbf{A}$

$D_{2}=\mathbf{P}_{1}(t) \mathbf{X}(t) \mathbf{T}(t-\tau(t)) \mathbf{B H A}$

$D_{3}=\mathbf{L}(t) \mathbf{C A}$

$I=\mathbf{X}(t) \mathbf{K Q}(t) \mathbf{H A}$.

So that, Eq. (1) can be represented by the following matrix equation as where

$\mathbf{T}(-\tau(t))=\left[\begin{array}{cccc}\left(\begin{array}{l}0 \\ 0\end{array}\right)(-\tau(t))^{0} & \left(\begin{array}{l}1 \\ 0\end{array}\right)(-\tau(t))^{1} & \ldots & \left(\begin{array}{c}N \\ 0\end{array}\right)(-\tau(t))^{N} \\ 0 & \left(\begin{array}{l}1 \\ 1\end{array}\right)(-\tau(t))^{0} & \ldots & \left(\begin{array}{c}N \\ 1\end{array}\right)(-\tau(t))^{N-1} \\ \vdots & \vdots & \ddots & \vdots \\ 0 & 0 & \cdots & \left(\begin{array}{c}N \\ N\end{array}\right)(-\tau(t))^{0}\end{array}\right]$.

Diversely, the kernel function $K(t, s)$ is obtained by using the Taylor series as

$K(t, s)=\sum_{i, j=0}^{N} k_{i j} t^{i} s^{j}, k_{i j}=\frac{1}{i ! j !} \frac{\partial^{i+j} K(0,0)}{\partial t^{i} \partial s^{j}}, K=\left[k_{i j}\right], i, j=0,1, \ldots, N$.

Then, its matrix form is written as

$\mathbf{K}(t, s)=\mathbf{X}(t) \mathbf{K} \mathbf{X}^{T}(s)$.

$$
\begin{aligned}
& \mathbf{L}(t) \mathbf{C A}+\mathbf{P}_{1}(t) \mathbf{X}(t) \mathbf{T}(t-\tau(t)) \mathbf{B H A} \\
& \quad=\mathbf{P}_{0}(t) \mathbf{L}(t) \mathbf{A}+\mathbf{X}(t) \mathbf{K} \mathbf{Q}(t) \mathbf{H A}+\mathbf{G}(t) .
\end{aligned}
$$

\section{Method of solution}

In this section, the collocation points are defined by

$t_{i}=\frac{b}{N} i, \quad i=0,1, \ldots, N$

Thus, the collocation points (17) are substituted into Eq. (16) and the fundamental matrix is obtained asor

$$
\begin{aligned}
& \mathbf{L}\left(t_{i}\right) \mathbf{C A}+\mathbf{P}_{1}\left(t_{i}\right) \mathbf{X}\left(t_{i}\right) \mathbf{T}\left(t_{i}-\tau\left(t_{i}\right)\right) \mathbf{B H A} \\
& \quad=\mathbf{P}_{0}\left(t_{i}\right) \mathbf{L}\left(t_{i}\right) \mathbf{A}+\mathbf{X}\left(t_{i}\right) \mathbf{K} \mathbf{Q}\left(t_{i}\right) \mathbf{H A}+\mathbf{G}\left(t_{i}\right)
\end{aligned}
$$




$$
\left\{\mathbf{L C}+\mathbf{P}_{1} \mathbf{X T}_{\tau} \mathbf{B H}-\mathbf{P}_{0} \mathbf{L}-\mathbf{X K Q H}\right\} \mathbf{A}=\mathbf{G}
$$

where

$$
\mathbf{L}=\left[\begin{array}{cccc}
L_{0}\left(t_{0}\right) & L_{1}\left(t_{0}\right) & \ldots & L_{N}\left(t_{0}\right) \\
L_{0}\left(t_{1}\right) & L_{1}\left(t_{1}\right) & \ldots & L_{N}\left(t_{1}\right) \\
\vdots & \vdots & \ddots & \vdots \\
L_{0}\left(t_{N}\right) & L_{1}\left(t_{N}\right) & \ldots & L_{N}\left(t_{N}\right)
\end{array}\right], \quad \mathbf{A}=\left[\begin{array}{c}
a_{0} \\
a_{1} \\
\vdots \\
a_{N}
\end{array}\right], \mathbf{G}=\left[\begin{array}{c}
g\left(t_{0}\right) \\
g\left(t_{1}\right) \\
\vdots \\
g\left(t_{N}\right)
\end{array}\right],
$$

$\mathbf{P}_{0}=\operatorname{diag}\left[P_{0}\left(t_{0}\right) P_{0}\left(t_{1}\right) \ldots P_{0}\left(t_{N}\right)\right]$,

$\mathbf{P}_{1}=\operatorname{diag}\left[P_{1}\left(t_{0}\right) P_{1}\left(t_{1}\right) \ldots P_{1}\left(t_{N}\right)\right]$,

$\mathbf{X}=\operatorname{diag}\left[\begin{array}{lll}X\left(t_{0}\right) & X\left(t_{1}\right) \ldots X\left(t_{N}\right)\end{array}\right]$,

$\mathbf{T}_{\tau}=\operatorname{diag}\left[X\left(t-\tau\left(t_{0}\right)\right) X\left(t-\tau\left(t_{1}\right)\right) \ldots X\left(t-\tau\left(t_{N}\right)\right)\right]$.

Briefly,

$$
\mathbf{W A}=\mathbf{G} \text { or }[\mathbf{W} ; \mathbf{G}]
$$

Here we have the augmented matrix form of Eq. (18). Besides, we consider the initial condition which is given in Eq. (2). Its matrix form is defined in Eq. (15). By putting the row from (15) in place of the last row of (19),

$\tilde{\mathbf{W}}=\left[\begin{array}{ccccc}\omega_{00} & \omega_{01} & \omega_{02} & \ldots & \omega_{0 N} \\ \omega_{10} & \omega_{11} & \omega_{12} & \ldots & \omega_{1 N} \\ \vdots & \vdots & \vdots & \ddots & \vdots \\ \omega_{(N-1) 0} & \omega_{(N-1) 1} & \omega_{(N-1) 2} & \ldots & \omega_{(N-1) N} \\ u_{00} & u_{01} & u_{02} & \ldots & u_{0 N}\end{array}\right], \quad \tilde{\mathbf{G}}=\left[\begin{array}{c}g\left(t_{0}\right) \\ g\left(t_{1}\right) \\ \vdots \\ g\left(t_{N-1}\right) \\ \lambda_{0}\end{array}\right]$.

Then, we construct the new augmented matrix as

$\tilde{\mathbf{W}} \mathbf{A}=\tilde{\mathbf{G}}$ or $[\tilde{\mathbf{W}} ; \tilde{\mathbf{G}}]$.

In Eq. (20), if $\operatorname{rank}[\tilde{\mathbf{W}}]=\operatorname{rank}[\tilde{\mathbf{W}} ; \tilde{\mathbf{G}}]=N+1$, then the coefficients matrix $\mathbf{A}$ is uniquely determined with the help of Gauss Elimination procedure [15-22]. Then, by using Eq. (3), the problem (1)-(2) is solved numerically and its numerical solution is obtained as in the form

$y(t) \cong y_{N}(t)=\sum_{n=0}^{N} a_{n} L_{n}(t)$.

\section{Analysis of the method}

In this section, some properties of the method are introduced. The stability of the collocation methods has been investigated previously by Brunner et al. [23, 24]. Besides, the stability of the related problem has been also considered by some authors $[25,26]$. However, existence and uniqueness theorems and convergence of the method are given as follows.

\section{Convergence of the method}

Definition 1 Let $y_{N}(t)$ be the approximate solution of the problem (1)-(2) which has an exact solution as $y(t)$. Then, the collocation method is said to be convergent if an only if

$\lim _{h \rightarrow 0}\left|y_{N}\left(t_{i}\right)-y\left(t_{i}\right)\right| \rightarrow 0, \quad i=0,1, \ldots, N$,

where $h$ is the step size and $h \rightarrow 0, N \rightarrow \infty$ [27-31].

Definition 2 If the largest number is $p$ for the finite constant $C$, then the method is of order $p$ such that [27-31]

$\left|y_{N}\left(t_{i}\right)-y\left(t_{i}\right)\right| \leq C h^{p}, \quad i=0,1, \ldots, N$.

where $h$ is the step size and $h \rightarrow 0, N \rightarrow \infty$ [27-29].

Theorem 1 Consider the first-order integro-differential equation including neutral terms and variable delays in $E q$. (1) under the initial condition (2). Then,

i. $g(t)$ is continuous in $0 \leq t \leq b$,

ii. $K(t, s)$ is a continuous function for $0 \leq t \leq b$ and $\|y\|<\infty$,

iii. $\quad K(t, s)$ satisfies a Lipschitz condition as follows

$$
\left\|K(t, s) y_{1}-K(t, s) y_{2}\right\| \leq L\left\|y_{1}-y_{2}\right\|
$$

for all $0 \leq t, s \leq b$. Then, the problem (1)-(2) has a unique solution [27-31].

Theorem 2 Consider that $g(t) \in C\left[I \times \mathbb{R}^{N}, \mathbb{R}^{N}\right]$, $K(t, s) \in C\left[\begin{array}{c}C-v(t) \\ I\end{array} I \times \mathbb{R}^{N}, \mathbb{R}^{N}\right]$ and $K(t, s) \in C\left[I \times I \times \mathbb{R}^{N}, \mathbb{R}^{N}\right]$ for $\quad \int_{t-u(t)}|K(t, s) y(s)| d s \leq N \quad$ for $\quad 0 \leq t, s \leq b$, $y \in \Omega=\left\{\phi \in C\left[I, \mathbb{R}^{N}\right]: \phi_{0}(0)=t_{0}\right.$ and $\left.\left|\phi(x)-\lambda_{0}\right| \leq b\right\}$. Then, our initial value problem (IVP) (1)-(2) has at least one solution [27-30].

\section{Existence and uniqueness}

Theorem 3 Consider the first-order integro-differential equation including neutral terms and variable delays in Eq. (1) under the initial condition (2). Assume that $g(t)$ and $K(t, s)$ are continuous functions which satisfy the Lipschitz conditions. Then,

$$
\begin{aligned}
& \left\|g(t) y_{1}-g(t) y_{2}\right\| \leq L\left\|y_{1}-y_{2}\right\|, \\
& \left\|K(t, s) y_{1}-K(t, s) y_{2}\right\| \leq L\left\|y_{1}-y_{2}\right\|,
\end{aligned}
$$


for every $\left|t-t_{0}\right| \leq a$ and $\left|s-t_{0}\right| \leq a$ for any initial value $t_{0}$ and the positive constant $a>0,\left\|y_{1}\right\|<\infty$ and $\left\|y_{2}\right\|<\infty$. Then, the IVP has a unique solution.

\section{Error analysis}

In this section, we investigate the absolute error function $E_{N}(t)$ for $t=t_{p} \in[a, b], \quad p=0,1,2, \ldots$.

$E_{N}\left(t_{p}\right)=\left|y_{N}\left(t_{p}\right)-y^{\prime}\left(t_{p}\right)-P_{1}\left(t_{p}\right) y^{\prime}\left(t_{p}-\tau\left(t_{p}\right)\right)+P_{0}\left(t_{p}\right) y\left(t_{p}\right)+\int_{t-u(t)}^{t-v(t)} K\left(t_{p}, s\right) y(s) \mathrm{d} s\right| \cong 0$

Proof [28-30].

\section{Accuracy}

In this section, the accuracy of the approximate solution is investigated. As an important factor for the numerical methods in the literature, the approximate solutions are corrected with regard to the residual error correction procedure. On the other hand, a brief error analysis is given in order to reach the approximation for the problem (1)-(2).

\section{Residual correction}

In here, the residual correction is given in order to improve the solutions and for the comprehensive error analysis for the approximate solution of the problem (1)-(2) [17, 18, 32-37]. and the accuracy of the numerical solutions is checked. Specifically, if $E_{N}\left(t_{q}\right) \leq 10^{-k_{q}}\left(k_{q}\right.$ any positive integer) is small enough, then the approximation has its reliability.

\section{Algorithm}

In here, the present method is shown by its algorithm. The steps are explained clearly in order to see the implementation of the computer programming part of the work.

Step 0

Step 1

Step 2
Input initial data: $P_{0}(t), P_{1}(t), u(t)$, $v(t)$ and $\tau(t)$

Set $m \leq N$ for $m \in \mathbb{N}$

Construct the matrices such as $\mathbf{L}(t), \mathbf{B}, \mathbf{H}$

$R_{N}(t)=\left|y_{N}^{\prime}(t)+P_{1}(t) y_{N}^{\prime}(t-\tau(t))-P_{0}(t) y_{N}(t)-\int_{t-u(t)}^{t-v(t)} K(t, s) y_{N}(s) \mathrm{d} s\right|, \quad y^{\prime}(0)=\lambda_{0}$.

Now, let us consider the error function as Step 3 Replace in the fundamental equa$e_{N}(t)=y(t)-y_{N}(t)$. Then, we construct an error problem in the form

$e_{N}^{\prime}(t)+P_{1}(t) e_{N}^{\prime}(t-\tau(t))=P_{0}(t) e_{N}(t)+\int_{t-u(t)}^{t-v(t)} K(t, s) e_{N}(s) \mathrm{d} s, \quad e_{N}^{\prime}(0)=0$.

Subsequently, the numerical method is applied on the error problem in Eq. (26) and we have the approximate solution for the error function in the form as follows

$e_{N, M}(t)=\sum_{n=0}^{M} a_{n}^{*} L_{n}(t)$

Thus, the corrected approximate solution is obtained as

$$
y_{N, M}(t)=y_{N}(t)+e_{N, M}(t) \text {. }
$$

Step 4

Step 5

Step 6

Step 7

Step 8

Step 9
Put the collocation points, $t_{i}=\frac{b}{N} i, \quad i=0,1, \ldots, N$ into the fundamental equation in $\mathrm{S} 3$

Calculate $[\mathbf{W} ; \mathbf{G}]$

Compute the matrix for the initial condition

Substitute the outcome from S6 into the matrix in $\mathrm{S} 5$ and get $[\tilde{\mathbf{W}} ; \tilde{\mathbf{G}}]$

Determine the system in S7.

Output: $y_{N}(t)$

Accuracy check: $E_{N}(t)$ 
Table 1 Error comparison for different truncation values: $N=6,8$ and 10 for $0 \leq t \leq 1$ of Example 2 with $\lambda=2$

\begin{tabular}{llll}
\hline$t$ & $E_{6}(t)$ & $E_{8}(t)$ & $E_{10}(t)$ \\
\hline 0.0 & $0.310003 \mathrm{E}-07$ & $0.152872 \mathrm{E}-09$ & $0.7290084 \mathrm{E}-10$ \\
0.1 & $0.362380 \mathrm{E}-07$ & $0.182554 \mathrm{E}-09$ & $0.8628143 \mathrm{E}-10$ \\
0.2 & $0.392103 \mathrm{E}-07$ & $0.179427 \mathrm{E}-09$ & $0.1005388 \mathrm{E}-10$ \\
0.3 & $0.452390 \mathrm{E}-08$ & $0.179520 \mathrm{E}-09$ & $0.1167447 \mathrm{E}-10$ \\
0.4 & $0.460112 \mathrm{E}-08$ & $0.748210 \mathrm{E}-10$ & $0.1371187 \mathrm{E}-11$ \\
0.5 & $0.470210 \mathrm{E}-08$ & $0.739810 \mathrm{E}-10$ & $0.1634357 \mathrm{E}-11$ \\
0.6 & $0.469890 \mathrm{E}-08$ & $0.741415 \mathrm{E}-10$ & $0.2083742 \mathrm{E}-11$ \\
0.7 & $0.467535 \mathrm{E}-08$ & $0.741883 \mathrm{E}-10$ & $0.3533296 \mathrm{E}-11$ \\
0.8 & $0.480254 \mathrm{E}-08$ & $0.741851 \mathrm{E}-10$ & $0.9147544 \mathrm{E}-11$ \\
0.9 & $0.479244 \mathrm{E}-08$ & $0.758964 \mathrm{E}-10$ & $0.2819447 \mathrm{E}-11$ \\
1.0 & $0.365974 \mathrm{E}-07$ & $0.120792 \mathrm{E}-09$ & $0.8338398 \mathrm{E}-10$ \\
\hline
\end{tabular}

Step 10

If $E_{N}\left(t_{q}\right) \cong 0$. Stop

Step 11

Else back $\mathrm{S} 1$

\section{Numerical experiments}

Numerical experiments section gives us an idea about the method and its applicability, validity and reliability. The applications of this method have been implemented by using some numerical illustrations. Here Maple and MATLAB computer programs are used for the calculation algorithm and plotting.

Example 1 Firstly, the first-order Volterra integro-differential equation including neutral term and variable delay.

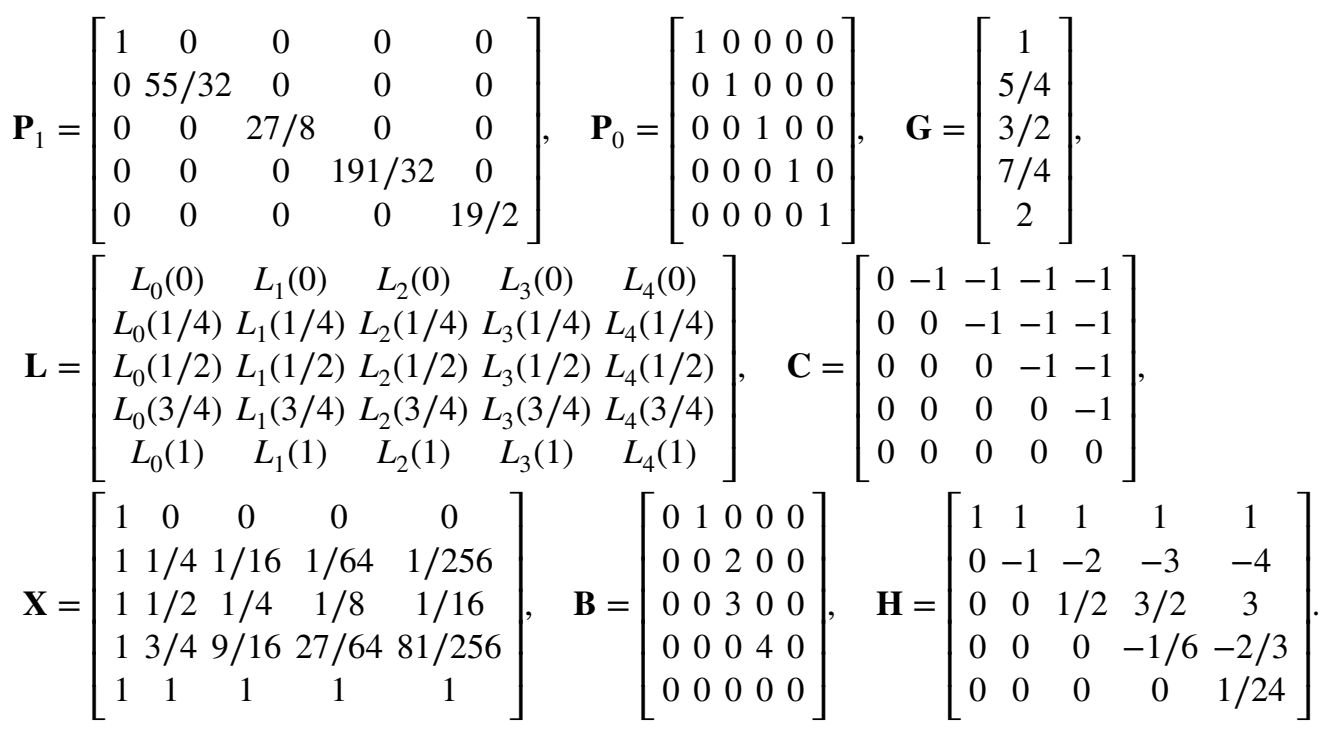


Table 3 Error comparison for different truncation values: $N$ $=5,6,8$ and 10 for $0 \leq t \leq 1$ of Example 3

\begin{tabular}{lllll}
\hline \multicolumn{5}{l}{ Laguerre collocation method (LCM) error results } \\
\hline$t$ & $E_{5}(t)$ & $E_{6}(t)$ & $E_{8}(t)$ & $E_{10}(t)$ \\
\hline 0.0 & $0.1592426 \mathrm{E}-04$ & $0.1460124 \mathrm{E}-05$ & $0.1259787 \mathrm{E}-07$ & $0.7290084 \mathrm{E}-09$ \\
0.1 & $0.3395409 \mathrm{E}-04$ & $0.3266380 \mathrm{E}-05$ & $0.1484878 \mathrm{E}-07$ & $0.8628143 \mathrm{E}-09$ \\
0.2 & $0.7241097 \mathrm{E}-04$ & $0.3894070 \mathrm{E}-05$ & $0.1739167 \mathrm{E}-07$ & $0.1005388 \mathrm{E}-08$ \\
0.3 & $0.1162061 \mathrm{E}-03$ & $0.2697500 \mathrm{E}-05$ & $0.2016250 \mathrm{E}-07$ & $0.1167447 \mathrm{E}-08$ \\
0.4 & $0.1355990 \mathrm{E}-03$ & $0.8985236 \mathrm{E}-06$ & $0.2274026 \mathrm{E}-06$ & $0.1371187 \mathrm{E}-08$ \\
0.5 & $0.8420424 \mathrm{E}-04$ & $0.2892807 \mathrm{E}-05$ & $0.2366310 \mathrm{E}-06$ & $0.1634357 \mathrm{E}-08$ \\
0.6 & $0.1030002 \mathrm{E}-03$ & $0.1772840 \mathrm{E}-04$ & $0.1910115 \mathrm{E}-06$ & $0.2083742 \mathrm{E}-08$ \\
0.7 & $0.5116663 \mathrm{E}-03$ & $0.6075665 \mathrm{E}-04$ & $0.5844883 \mathrm{E}-06$ & $0.3533296 \mathrm{E}-08$ \\
0.8 & $0.1250068 \mathrm{E}-02$ & $0.1554554 \mathrm{E}-03$ & $0.4857541 \mathrm{E}-05$ & $0.9147544 \mathrm{E}-08$ \\
0.9 & $0.2451094 \mathrm{E}-02$ & $0.3354244 \mathrm{E}-03$ & $0.1585916 \mathrm{E}-05$ & $0.2819447 \mathrm{E}-07$ \\
1.0 & $0.4274240 \mathrm{E}-02$ & $0.6465534 \mathrm{E}-03$ & $0.3807165 \mathrm{E}-05$ & $0.8338398 \mathrm{E}-07$ \\
\hline
\end{tabular}

So, the augumented matrix is found as

$$
[\mathrm{W} ; \mathrm{G}]=\left[\begin{array}{cccccccc}
2 / 5 & -1 / 5 & 0 & 0 & 0 & ; & 1 \\
1 / 2 & -1 / 4 & 0 & 0 & 0 & ; & 5 / 4 \\
3 / 5 & -3 / 10 & 0 & 0 & 0 & ; & 3 / 2 \\
7 / 10 & -7 / 20 & 0 & 0 & 0 & ; & 7 / 4 \\
4 / 5 & -2 / 5 & 0 & 0 & 0 & 0 & 2
\end{array}\right] .
$$

Now, the initial condition is defined in (31) has the matrix form as

$\left[y^{\prime}(0)\right]=\left[\begin{array}{lllll}1 & 0 & 0 & 0 & 0\end{array}\right]$.

So that the new augmented matrix is constructed as

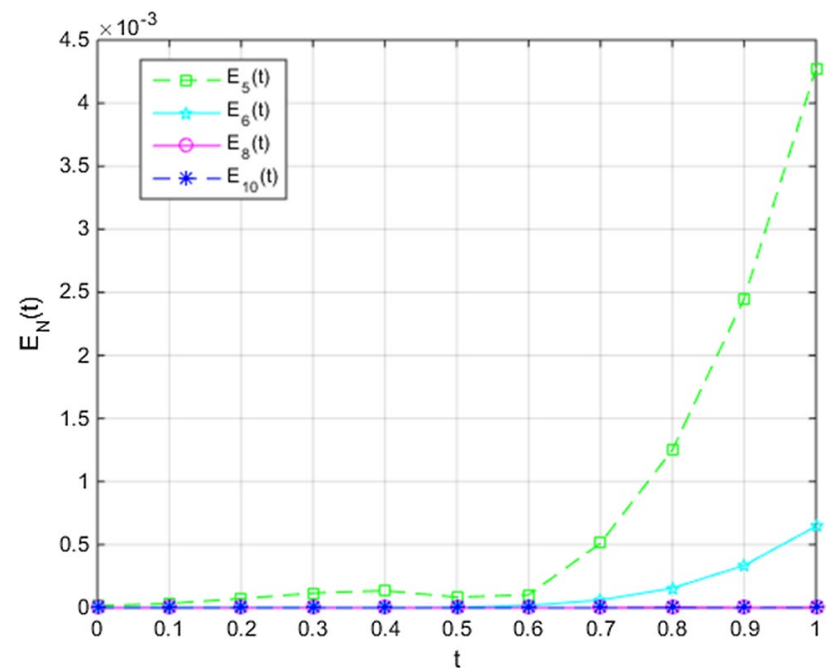

Fig. $2 E_{N}(t)$ error comparison for Example 2
$[\tilde{\mathrm{W}} ; \tilde{\mathrm{G}}]=\left[\begin{array}{ccccccc}2 / 5 & -1 / 5 & 0 & 0 & 0 & ; & 1 \\ 1 / 2 & -1 / 4 & 0 & 0 & 0 & ; & 5 / 4 \\ 3 / 5 & -3 / 10 & 0 & 0 & 0 & ; & 3 / 2 \\ 7 / 10 & -7 / 20 & 0 & 0 & 0 & 0 & 7 / 4 \\ 1 & 0 & 0 & 0 & 0 & 0 & 0\end{array}\right]$

By following the procedure, the system is solved, and then, $a_{n}$ Laguerre coefficients are found as follows

$a_{0}=2, \quad a_{1}=-1, \quad a_{2}=0, \quad a_{3}=0, \quad a_{4}=0$.

Consequently, these calculated coefficients are established and they are replaced in Eq. (32). Then, the exact solution of (30)-(31) is acquired as

$y(t)=t+1$.

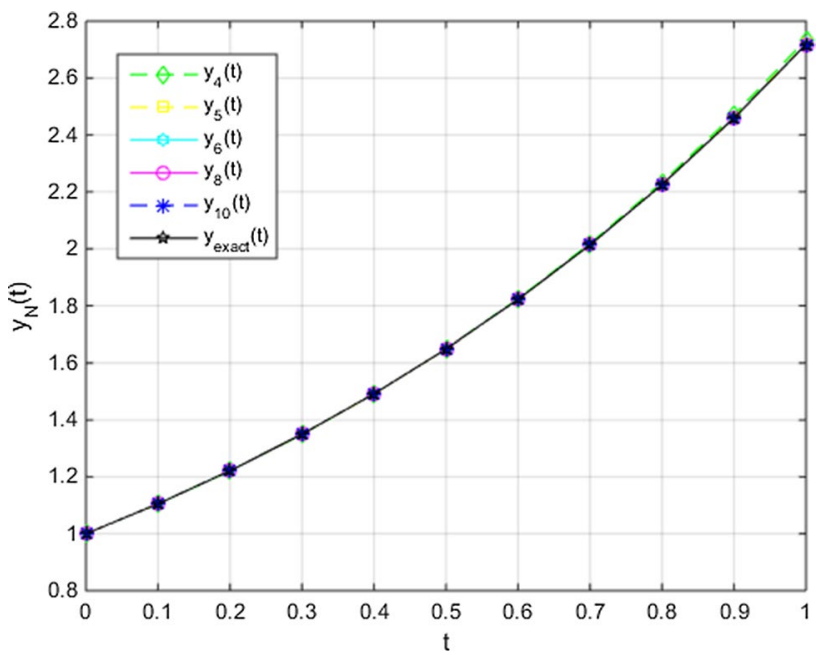

Fig. 3 Comparison between exact solution with the numerical solutions with regard to Laguerre approach for $N=4,5,6,8$ and 10 in Example 3 
Table 4 Error functions for different iterations with improved results of Example 3

\begin{tabular}{llll}
\hline$t$ & $e_{5,6}(t)$ & $e_{6,7}(t)$ & $e_{8,9}(t)$ \\
\hline 0.0 & $0.1942352 \mathrm{E}-07$ & $0.2450211 \mathrm{E}-08$ & $0.3221422 \mathrm{E}-09$ \\
0.1 & $0.1252100 \mathrm{E}-07$ & $0.2451328 \mathrm{E}-08$ & $0.3225781 \mathrm{E}-09$ \\
0.2 & $0.1278830 \mathrm{E}-07$ & $0.2481209 \mathrm{E}-08$ & $0.3236841 \mathrm{E}-09$ \\
0.3 & $0.1952003 \mathrm{E}-08$ & $0.3709150 \mathrm{E}-09$ & $0.3239577 \mathrm{E}-09$ \\
0.4 & $0.1955221 \mathrm{E}-08$ & $0.3719104 \mathrm{E}-09$ & $0.2715481 \mathrm{E}-10$ \\
0.5 & $0.1825573 \mathrm{E}-08$ & $0.3718551 \mathrm{E}-09$ & $0.2716773 \mathrm{E}-10$ \\
0.6 & $0.1821335 \mathrm{E}-08$ & $0.3798133 \mathrm{E}-09$ & $0.2720932 \mathrm{E}-10$ \\
0.7 & $0.1865131 \mathrm{E}-08$ & $0.3689412 \mathrm{E}-09$ & $0.2810013 \mathrm{E}-10$ \\
0.8 & $0.1033571 \mathrm{E}-07$ & $0.3690155 \mathrm{E}-09$ & $0.2812005 \mathrm{E}-10$ \\
0.9 & $0.1053218 \mathrm{E}-07$ & $0.2954771 \mathrm{E}-08$ & $0.3554127 \mathrm{E}-09$ \\
1.0 & $0.2495782 \mathrm{E}-07$ & $0.2961238 \mathrm{E}-08$ & $0.3578220 \mathrm{E}-09$ \\
\hline
\end{tabular}

Example 2 As a second example, the first-order Volterra integro-differential equation including neutral term and delay is considered [37]

$y^{\prime}(t)=(\lambda-1) \mathrm{e}^{(1-t)}-(\lambda+1) y(t)+y(t-1)-\lambda \int_{t-1}^{t} y(s) \mathrm{d} s, \quad t \geq 0$,

and the initial condition

$y(0)=1$,

corresponding to the exact solution $y_{\text {exact }}(t)=e^{t}$. Correspondingly, the problem (34)-(35) is solved by the similar approach which is followed in Example 1. In Table 1, absolute errors for different $N$ truncation values are demonstrated for their comparison. Herein this error comparison with different numerical methods: Laguerre collocation method (LCM) for $M=10$ and $N=9$ and mixed spline/spectral method (MSSM) with $h=0.2$ in Table 2 and in Fig. 1. From these comparisons, we can see apparently that more suitable and efficient results are obtained for the smaller when we have increasing $N$ value.

Example 3 Consider the first-order Volterra integro-differential equation including neutral term and delay from Eq. (1) with $P_{1}(t)=v(t)=g(t)=0, K(t, s)=u(t)=1$ and including a delay term $y(t-1)$ together with $P_{0}(t)=1$. Moreover, the initial condition is given as $y(0)=1$ and the exact solution is $y_{\text {exact }}(t)=e^{t}$. Then, the absolute errors for different $N$ truncation values are demonstrated for their comparison in Table 3 and Fig. 2. Besides, we can see the numerical solutions with regard to Laguerre approach and exact solution of the problem in Fig. 3. From these comparisons, we can see apparently that more suitable and efficient results are obtained for the smaller when we have increasing $N$ value. Subsequently, there are improved results with related to the
Sect. 4.1. Residual correction helps for a better approximation and give smaller values for the error function which is shown in Table 4.

\section{Conclusion}

In this study, a powerful numerical technique to determine the numerical solutions of first-order integro-differential equations including neutral terms and variable delays is proposed. The technique affords approximate solutions of the problem which are mainly close enough to the exact solutions with respect to $N$. Accuracy and applicability of the method have been proved by the visible results at the tables and the figures. The main advantages of this technique are including but not limited to straightforward coding, its apparent algorithm and accessible matrix calculations. Moreover, the error analysis including the residual correction supports the results with the additional error problem solution which is explained and applied in an example.

As a future outlook, this numerical study and the technique can be extended to other models with related to Volterra integro-differential equations including retarded term. However, some modifications are required [38].

Funding Open Access funding enabled and organized by Projekt DEAL.

Open Access This article is licensed under a Creative Commons Attribution 4.0 International License, which permits use, sharing, adaptation, distribution and reproduction in any medium or format, as long as you give appropriate credit to the original author(s) and the source, provide a link to the Creative Commons licence, and indicate if changes were made. The images or other third party material in this article are included in the article's Creative Commons licence, unless indicated otherwise in a credit line to the material. If material is not included in the article's Creative Commons licence and your intended use is not permitted by statutory regulation or exceeds the permitted use, you will need to obtain permission directly from the copyright holder. To view a copy of this licence, visit http://creativecommons.org/licenses/by/4.0/.

\section{References}

1. Bellen, A., Zennaro, M.: Numerical Methods for Delay Differential Equations. Oxford University Press (2013)

2. Kolmanovskii, V., Myshkis, A.: Applied Theory of Functional Differential Equations. Springer, Dordrecht (1992)

3. Godin, E.A., Kolmanovskii, V.B., Stengold, ESh.: Hypertension Disease as a Result of Altered Stress-Strained Vascular State. Nauka, Moscow (1990).. ((In Russian))

4. Brunner, H.: The numerical analysis of functional integral and integro-differential equations of Volterra type. Acta Numer. 13, 55 (2004)

5. Ming, W., Huang, C.: Collocation methods for Volterra functional integral equations with non-vanishing delays. Appl. Math. Comput. 296(2017), 198-214 (2017) 
6. Rawashdeh, E.A.: Numerical treatment of neutral fractional Volterra integro-differential equations with infinite delay. Italian J. Pure Appl. Math. 37(2017), 89-96 (2017)

7. Rashed, M.T.: Numerical solution of functional differential integral and integro-differential equations. Appl. Math. Comput. 156(2004), 485-492 (2004)

8. Jackiewicz, Z.: The numerical solution of Volterra functional differential equations of neutral type. SIAM J. Numer. Anal. 18(4), 615-626 (1981)

9. Reutskiy, SYu.: The backward substitution method for multipoint problems with linear Volterra-Fredholm integro-differential equations of the neutral type. J. Comput. Appl. Math. 296(2016), 724 738 (2016)

10. Liu, Y.: Numerical solution of implicit neutral functional differential equations. SIAM J. Numer. Anal. 36(2), 516-528 (1999)

11. Enright, W.H., Hu, M.: Continuous Runge-Kutta methods for neutral Volterra integro-differential equations with delay. Appl. Numer. Math. 24(2-3), 175-190 (1997)

12. Koto, T.: Stability of Runge-Kutta methods for delay integrodifferential equations. J. Comput. Appl. Math. 145(2), 483-492 (2002)

13. Sedaghat, S., Ordokhani, Y., Dehghan, M.: On spectral method for Volterra functional integro-differential equations of neutral type. Numer. Funct. Anal. Optim. 35(2), 223-239 (2014)

14. Gürbüz, B., Sezer, M.: Modified Laguerre collocation method for solving 1-dimensional parabolic convection-diffusion problems. Math. Methods Appl. Sci. 41, 1-7 (2017)

15. Özel, M., Tarakçı, M., Sezer, M.: A numerical approach for a nonhomogeneous differential equation with variable delays. Math. Sci. 12(2), 145-155 (2018)

16. Yüzbaşı, Ş: Laguerre approach for solving pantograph-type Volterra integro-differential equations. Appl. Math. Comput. 232, 1183-1199 (2014)

17. Gürbüz, B., Sezer, M.: An hybrid numerical algorithm with error estimation for a class of functional integro-differential equations. Gazi Univ. J. Sci. 29(2), 419-434 (2016)

18. Gürbüz, B., Sezer, M., Güler, C.: Laguerre collocation method for solving Fredholm integro-differential equations with functional arguments. J. Appl. Math. 2014, 1-12 (2014)

19. Gürbüz, B., Sezer, M.: Modified operational matrix method for second-order nonlinear ordinary differential equations with quadratic and cubic terms. Int. J. Optim. Control Theor. Appl. (IJOCTA) 10(2), 218-225 (2020)

20. Gürbüz, B.: A novel method for solving a class of functional differential equations. Balıkesir Üniversitesi Fen Bilimleri Enstitüsü Dergisi 22(1), 66-79 (2020)

21. Gürbüz, B., Sezer, M.: Laguerre matrix-collocation method to solve systems of pantograph type delay differential equations. In: International Conference on Computational Mathematics and Engineering Sciences. Springer, Cham, pp. 121-132 (2019)

22. Gökmen, E., Gürbüz, B., Sezer, M.: A numerical technique for solving functional integro-differential equations having variable bounds. Comput. Appl. Math. 37(5), 5609-5623 (2018)
23. Brunner, H., Liang, H.: Stability of collocation methods for delay differential equations with vanishing delays. BIT Numer. Math. 50(4), 693-711 (2010)

24. Brunner, H.: The numerical solution of weakly singular Volterra functional integro-differential equations with variable delays. Commun. Pure Appl. Anal. 5(2), 261 (2006)

25. Zhao, J.J., Xu, Y., Liu, M.Z.: Stability analysis of numerical methods for linear neutral Volterra delay-integro-differential system. Appl. Math. Comput. 167(2), 1062-1079 (2005)

26. Rihan, F.A., Doha, E.H., Hassan, M.I., Kamel, N.M.: Numerical treatments for Volterra delay integro-differential equations. Comput. Methods Appl. Math. 9(3), 292-318 (2009)

27. Jhinga, A., Patade, J., Daftardar-Gejji, V.: Solving Volterra Integro-Differential Equations involving Delay: A New Higher Order Numerical Method. arXiv preprint (2020).

28. Patade, J., Bhalekar, S.: A novel numerical method for solving Volterra integro-differential equations. Int. J. Appl. Comput. Math. 6(1), 1-19 (2020)

29. Linz, P.: A method for solving nonlinear Volterra integral equations of the second kind. Math. Comput. 23(107), 595-599 (1969)

30. Lakshmikantham, V., Rao, M.R.M.: Theory of Integro-Differential Equations. Gordon and Breach Science Publishers, Amsterdam (1995)

31. Wang, K., Wang, Q.: Taylor collocation method and convergence analysis for the Volterra-Fredholm integral equations. J. Comput. Appl. Math. 260, 294-300 (2014)

32. Oliveira, F.A.: Collocation and residual correction. Numer. Math. 36(1), 27-31 (1980)

33. Dahm, J.P., Fidkowski, K.: Error estimation and adaptation in hybridized discontinuous Galerkin methods. In: 52nd Aerospace Sciences Meeting, p. 0078 (2014)

34. Ainsworth, M., Oden, J.T.: A unified approach to a posteriori error estimation using element residual methods. Numer. Math. 65(1), 23-50 (1993)

35. Braess, D., Schöberl, J.: Equilibrated residual error estimator for edge elements. Math. Comput. 77(262), 651-672 (2008)

36. Braess, D., Pillwein, V., Schöberl, J.: Equilibrated residual error estimates are p-robust. Comput. Methods Appl. Mech. Eng. 198(13-14), 1189-1197 (2009)

37. El-Hawary, H.M., El-Shami, K.A.: Numerical solution of Volterra delay-integro-differential equations via spline/spectral methods. Int. J. Differ. Equ. Appl. 12(3) (2013)

38. Hashemizadeh, E., Ebadi, M.A.: A numerical solution by alternative Legendre polynomials on a model for novel coronavirus (COVID-19). Adv. Diff. Equ. 2020(1), 1-12 (2020)

Publisher's Note Springer Nature remains neutral with regard to jurisdictional claims in published maps and institutional affiliations. 